\title{
General decay and blow-up results for a nonlinear pseudoparabolic equation with Robin-Dirichlet conditions
}

\author{
Le Thi Phuong Ngoc ${ }^{1}$, Nguyen Huu Nhan ${ }^{2}$, and Nguyen Thanh Long ${ }^{3}$ \\ ${ }^{1}$ University of Khanh Hoa \\ ${ }^{2}$ Nguyen Tat Thanh University \\ ${ }^{3}$ University of Science, Ho Chi Minh City, Vietnam,
}

September 24, 2020

\begin{abstract}
This paper is devoted to the study of a nonlinear pseudoparabolic equation in an annular with Robin-Dirichlet conditions. At first, by applying the standard Faedo-Galerkin method, we prove existence and uniqueness results. Next, using concavity method, we prove blow-up results for solutions when the initial energy is nonnegative or negative, then we also establish the lifespan for the equation via finding the upper bound and the lower bound for the blow-up times. Finally, we give a sufficient condition for the global existence and decay of weak solutions.
\end{abstract}

\section{Hosted file}

NgocNhanLong(SubMMAS) Sep23,2020.pdf available at https://authorea.com/users/360592/articles/ 482900-general-decay-and-blow-up-results-for-a-nonlinear-pseudoparabolic-equation-withrobin-dirichlet-conditions

\section{Hosted file}

NgocNhanLong(SubMMAS) Sep23,2020.tex available at https://authorea.com/users/360592/articles/ 482900-general-decay-and-blow-up-results-for-a-nonlinear-pseudoparabolic-equation-withrobin-dirichlet-conditions 